\title{
Thermodynamics, kinetics and reaction chemistry of aerobic respiration
}

\author{
Kelath Murali Manoj ${ }^{{ }^{*}} \&$ Nikolai Mikhailovich Bazhin ${ }^{2 *}$ \\ ${ }^{*}$ Satyamjayatu: The Science \& Ethics Foundation, \\ Snehatheeram, Kulappully, Shoranur-2 (PO), Kerala, India-679122. \\ satyamjayatu@yahoo.com; murman@ satyamjayatu.com \\ *2 Institute of Chemical Kinetics and Combustion \\ Russian Academy of Sciences, St. Institutskaya 3, 630090 Novosibirsk, Russia. \\ bazhin8999@kinetics.nsc.ru
}

\begin{abstract}
Three fundamental queries are addressed on the thermodynamics of aerobic cellular respiration (viz. ATP-synthesis and thermogenesis)- (1) Can the energy of oxygen reduction be utilized for proton transport?, (2) Is the trans-membrane proton differential harness-able as a potential energy capable of doing useful work?, and (3) If the movement of a miniscule amount of mitochondrial protons could give rise to a potential of $\sim 200 \mathrm{mV}$ and if such an electrical energy could sponsor ATP-synthesis. Further, we provide a definite answer to a fourth questionwhat is the thermodynamic role of protons in the oxygen-centric scheme of aerobic respiration? Finally, we demonstrate that the rotary ATPase activity of Complex $\mathrm{V}$ cannot account for physiological ATP synthesis and establish that the murburn reaction model explains the kinetics of mitochondrial oxidative phosphorylation.
\end{abstract}

Keywords: thermodynamics, kinetics, mitochondria, aerobic respiration, chemiosmosis, murburn concept 


\section{Introduction: The fundamental powering logic of life}

Life forms could be considered as "intelligent" machines or engines capable of doing useful work. In a classical combustion engine, a hydrocarbon fuel (like octane) is burnt using an oxidant (like oxygen). This liberates heat and/or expanding gases, which can be harnessed for commissioning work in real space and time. In most living beings, the potential energy to carry out several "non-spontaneous works of life" arises from the chemical energy reserve of ATP. Further, the kinetic viability of reactions is enhanced by heat generated within the living organism. Both the chemical energy reserve and thermal energy are primarily generated in the powerhouse of the cell, the mitochondria. The diverse organic molecules presented from the breakdown of food (derived from heterotrophic or autotrophic nutrition, like glucose and fatty/amino acids) serve as the biological fuel, which is used to make NADH. The metabolic process of NADH driven ATP-synthesis is mitochondrial oxidative phosphorylation (mOxPhos). It is also understood that the generation of heat (thermogenesis) within mitochondria is a consequence of mOxPhos. In this manuscript, we shall deal with the physical life-sustaining drives (mOxPhos and thermogenesis) from the physicist's perspective. For, no matter how elegant a biological explanation may be, it has to fit into the known laws of physics.

\section{Materials \& Methods: The prevailing textbook ideas on aerobic cellular respiration}

The parameters for ATP synthesis, proceeding from the readily available precursors in mitochondria, are calculated by (Alberty, 2003):

$$
\mathrm{ADP}-\mathrm{OH}+\mathrm{P}-\mathrm{OH}=\mathrm{ADP}-\mathrm{OP}+\mathrm{H}_{2} \mathrm{O} ; \Delta_{\mathrm{r}} G^{\mathrm{O}} \sim+36 \mathrm{~kJ} / \mathrm{mol}, \Delta_{\mathrm{r}} H^{\prime \mathrm{O}} \sim+26 \mathrm{~kJ} / \mathrm{mol} .
$$


Therefore, the process is both endergonic and endothermic. The ATP-synthesis or esterification reaction (1) is believed to be catalyzed by Complex V (which serves as a rotary ATPsynthase) via the simple equilibrium-driven equation (Nelson \& Cox, 2008)-

$$
\mathrm{K}_{\text {eq.ATPase }}=\left([\mathrm{ADP}-\mathrm{OP}] \cdot\left[\mathrm{H}_{2} \mathrm{O}\right]\right) /([\mathrm{ADP}-\mathrm{OH}] \cdot[\mathrm{P}-\mathrm{OH}])----(2)
$$

Currently, Mitchell's chemiosmosis is the popular explanation for mOxPhos (Nelson \& Cox, 2008). Per this understanding, the redox proteins located in the inner mitochondrial membrane serve as electron transfer agents, moving electrons along a definite and sequential route called Electron Transport Chain (ETC). One of these proteins is exemplified by Complex IV (cytochrome oxidase or Cox). Its reaction scheme (per the ETC understanding) is given below (Alberty, 2003):

$$
4 \text { Cytc-red }+8 \mathrm{H}^{+}{ }_{\text {in }}+\mathrm{O}_{2} \rightarrow 4 \text { Cytc-ox }+4 \mathrm{H}^{+} \text {out }+2 \mathrm{H}_{2} \mathrm{O} ; \Delta_{\mathrm{r}} G^{\prime \mathrm{o}} \sim-246 \mathrm{~kJ} / \mathrm{mol} \text {-- (3) }
$$

The yield of a reaction such as (2) is significantly higher than the energy required for the ATP synthesis reaction (1). According to the chemiosmotic explanation (Nelson \& Cox, 2008), the energy from the various redox reactions of ETC proteins (such as 2) is used to transport/pump protons from the mitochondrial matrix into the inter-membrane space (IMS), which requires $\sim 22$ $\mathrm{kJ} / \mathrm{mol}$. Further, such protons are believed to "retain or conserve" a significant amount of this energy too. That is- the chemical proton differential (or concentration gradient) and electrical potential gradient that supposedly results thereafter contains a usable potential energy. Though there is no formal thermodynamics-rooted and $a b$ inito based derivation for this statement, the popular "chemiosmotic energetics equation" is quantitatively expressed as:

$$
\Delta \mathrm{p}=(0.06) \Delta \mathrm{pH}+\Delta \Psi
$$

In the above equation, the left hand term of $\Delta \mathrm{p}$ is the proton motive force (pmf or transmembrane potential, TMP) driving ATP synthesis. This $\Delta \mathrm{p}$ is an additive function of the trans- 
membrane electrical potential $(\Delta \Psi)$ and the trans-membrane chemical $\mathrm{pH}$ differential $(\Delta \mathrm{pH})$. That is-

$$
\begin{aligned}
& \Delta \mathrm{G}_{\text {chem }}=n \mathrm{RT} \ln \left(\left[\mathrm{H}^{+}{ }_{\text {in }}\right] /\left[\mathrm{H}_{\text {out }}^{+}\right]\right)--------(5) \\
& \Delta \mathrm{G}_{\text {elect }}=z \mathrm{~F} \Delta \Psi ;\left(\text { where } \Delta \Psi=\Psi_{\text {in }}-\Psi_{\text {out }}\right) \text {-------- (6) }
\end{aligned}
$$

In the above equations, $n$ is the number of protons and $z$ is the formal charge on a proton (and R, T, F have their standard definitions/values). Substituting the known constants and physiological concentrations into equations (4) and (5), we get the approximate values of the three respective terms in equation (3) as $0.2 \mathrm{~V}, 0.17 \mathrm{~V}$ and $0.03 \mathrm{~V}$. It is therefore evident that the major contribution of proton motive force employed for ATP synthesis arises from the electrical component. Now, the actual ATP synthesis supposedly occurs in a second and sequential step, when the very same "energized" protons from a higher concentration regime pass through Complex V and return into the matrix. In this step, the protons serve as "triggering/activating" switch for Complex $\mathrm{V}$ and the proton gradient energy is expended on ATP synthesis. Under certain circumstances, the same proton gradient cum trans-membrane electrical potential (which shall henceforth be called as trans-membrane potential or TMP) is supposed to give thermogenesis. For example- in the presence of chemical uncouplers or active uncoupling proteins cum fatty acids, the $\Delta \mathrm{p}$ generated gets non-productively dissipated into heat (Manoj, 2018a). This is reasoned to occur because the pumped out protons (present in the IMS) re-enter matrix without going through Complex V. The utilization scheme of chemiosmotic TMP in mitochondrial respiration is shown in Figure 1. 

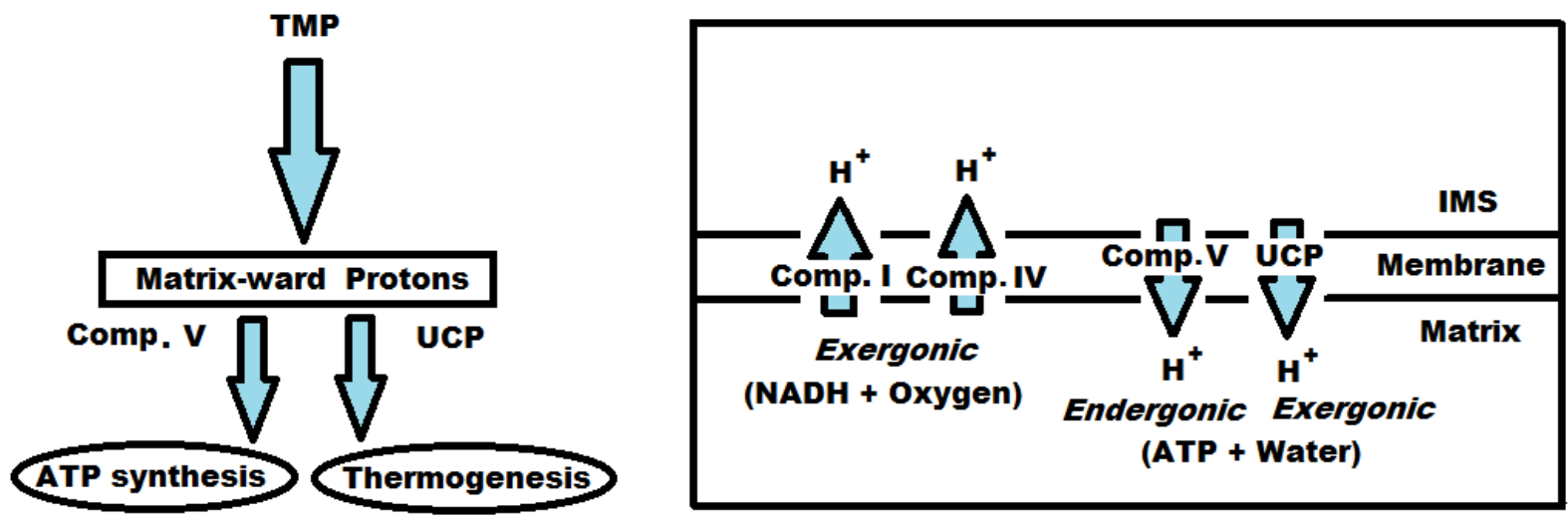

Figure 1: The "closed pot" chemiosmotic explanation for mitochondrial aerobic respiration: TMP (which is generated by trans-membrane proton-pumps like Comp. I \& Comp. IV) is the driving force for the matrix-ward movement of energized protons. Such "energized or crowded" protons within the IMS are either used for ATP synthesis (via Comp. V) or lost to thermogenesis (via $U C P$ ).

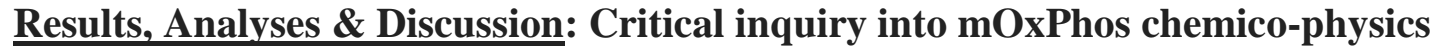

From the thermodynamic perspective, there are several fundamentally unacceptable issues with the chemiosmotic explanation. The arguments presented below are in conjunction and in agreement with the chemico-physical logic already detailed in our earlier works in this regard (Bazhin, 2015a,b; Bazhin, 2018; Manoj, 2017; Manoj, 2018a,b; Manoj et al., 2018, Manoj et al., 2019, Manoj, 2019).

Is it possible to utilize the energy of oxygen-reduction for proton-transport?

Let us assume that the complete reduction of a molecule of oxygen to two molecules of water is a highly viable reaction at Complex IV. In this reaction, the energy released translates on the degrees of freedom of the molecules - the products. Further, when interacting with the molecules 
within the milieu, this energy relaxes very quickly. The characteristic time scale lies in picosecond regimes and therefore, the energy relaxation rate constant is $\sim 10^{12} \mathrm{~s}^{-1}$ (Deng \& Stratt, 2002). Proton transport through the inner membrane with a thickness of about $>5 \mathrm{~nm}$ (Metzler, 2001) can occur with the help of some molecules, but only at the rate of diffusion velocity. If we take the diffusion coefficient $\sim 10^{-9} \mathrm{~m}^{2} / \mathrm{s}$ (Hubley et al., 1996), then the diffusion time runs to $\sim 10^{-7} \mathrm{~s}$. That is, the monomolecular transfer rate constant will be $\sim 10^{7} \mathrm{~s}^{-1}$. Then the efficiency (or part) of energy use for proton transport will be $10^{7} / 10^{12} \approx 10^{-5}$. Thus, the use of energy released during the reduction of oxygen for proton transport can only be a minute fraction of the total, and therefore, the process would be nonviable. Therefore, the chemiosmosis explanation is an invalid proposal from fundamental thermodynamics and kinetics perspective.

Can a proton concentration gradient created within "a closed Mitchellian mitochondrial operating system" be harnessed as a potential energy capable of doing useful work?

In biochemistry (Nelson \& Cox, 2008) it is generally accepted that Gibbs energy is real energy and can be used to produce useful work. However, some authors object to this statement (Strong \& Halliwell, 1970; Crabtree \& Taylor, 1979; Bazhin, 2015). The Gibbs energy only allows one to calculate the maximum useful work in a reversible process, and the source of energy in most cases is the heat of the environment (Bazhin, 2018). Violations of the second law of thermodynamics cannot and does not occur because concomitant with the useful work production, a chemical process takes place. Now, let's suppose that a system can produce protons' trans-membrane transport within a closed system for unknown reasons. As a result of proton transfer, the concentration of protons in the IMS would increase and this is accompanied 
by an increase in Gibbs energy (due to the work expended). In the chemiosmotic proposal, it is assumed that the work expended is converted into the potential energy of this system with the concomitant generation of a concentration gradient. However, this view is inconsistent with the known concepts of thermodynamics wherein it is a fundamental principle that ideal solutions of different concentrations do not have potential energy and there is no heat release with simple irreversible mixing (Prigogine \& Defay, 1954). Therefore, it follows that the work expended on creating any concentration gradient must be dissipated in the form of heat in the environment. As a result, protons passing or returning through Complex $\mathrm{V}$ cannot perform the work of the endergonic ATP synthesis. It should be noted that non-equilibrium systems (for example, a system with a concentration gradient), the processes which can occur with $\Delta G<0$ can be used to get a work (Bazhin, 2015). However, such systems must have the instruments to extract heat from the environment and use this heat to produce work. There is little evidence to say that Complex V has such a facet/attribute. Even if it so, that activity would not fall within the purview of Mitchell-Boyer explanation of ATP synthesis.

Can a miniscule amount of mitochondrial protons or their movements give rise to a TMP of -200 $\mathrm{mV}$ ? Can the electrical component of equation (5) provide energy for ATP synthesis?

In physiological state, the proton translocating chemical event leads to the potential contribution of only $0.03 \mathrm{mV}$. It is inexplicable with the chemiosmosis purview as to how the miniscule proton movement ( $\sim 6$ protons per mitochondrion at physiological state) would generate a surplus of $0.17 \mathrm{mV}$ electrical potential. The other organic and inorganic ions' strength is higher by at least six to seven orders of concentrations and the net charge afforded by protons is only a 
miniscule contribution of the overall electrostatics. The murburn chemical explanation is that this TMP arises due to the generation of negatively charged species formed within the matrix, as a result of one-electron or radical redox processes. Such species can have much higher concentrations (in decades of micromolar levels) and they are not directly "charge-neutralized" under the macroscopic purview of ionic equilibriums.

Let's assume that a charged species is moved from one macroscopic phase (say, matrix) to another macroscopic phase (say, IMS) and the two phases (which are charge-balanced within) are separated by a membrane. Then the TMP generated by charge translocation would only be a portion of the energy that would have been expended in the process that generated the TMP. Also, even if the required amount of protons are somehow produced in the system and pumped out, there is no mechanism known for the protons to retain or acquire a "high energy" status by crowding within the IMS. This is because the IMS is in equilibrium with cytoplasm. Further, in steady state, the electrical potential generated by trans-membrane activity can only be availed to do some useful work in another connected system, and it cannot be used to carry out work by moving the very protons back across the same membrane (Manoj, 2017). Furthermore, in an electric field, a proton can get/gain only kinetic energy. However, in a condensed medium, the kinetic energy will be dissipated to the environment in the form of heat during collisions with molecules. Therefore, an electrical component can at the most only provide only thermal energy in the mitochondria (Rubin, 2013) and this may only form more water (at the most!). However, there is little scope for this thermal energy to revert the catalytic functionality of Complex V, which is a natural ATPase (Manoj, 2018; Manoj et al., 2019a). As shown in Figure 1, it is not possible to theoretically justify how or why the protons' return to the matrix via Comp. V would 
support an endergonic process while the same process mediated by UCP is an exergonic process. In our earlier paper, we had shown that such perceptions are not only logically self-contradictory, they also flout the fundamental principles of thermodynamics (Manoj 2017). Further, we had discussed that the miniscule amounts of protons could not serve as triggers to the tens of thousands of Complex V per mitochondrion. We have also elaborated that in the physiological steady state, there is little scope for a TMP-based system to perform harness-able chemical work like ATP-synthesis. This is because -

A. There is no synchronization of the proton pumps (assuming that mitochondria generate protons for the same purpose in some unforeseen ways!) so that a high or steady TMP is generated.

B. There is no alternation or temporal change (of the magnitude or sign) of TMP so that Complex V may change its structure from an ATPase and become an ATPsynthase.

C. The structure of Complex V shows no components that could sense or tap such electric fieldchange and this protein has thermodynamics and kinetics terms supporting only an ATPase activity (and not ATPsynthase!) (Manoj et al., 2019a).

D. The closed pot perspective cannot explain the proton-deficient reaction scheme of mOxPhos wherein oxidation of NADH by oxygen gives water, which requires more protons than what is available within the matrix or IMS.

Can rotary ATPsynthase catalysis explain the kinetics and yield of mOxPhos?

Textbooks inform us that the value of equilibrium constant $\mathrm{K}_{\mathrm{eq}}$ for equation ( 1 or 2 ) is $\sim 5 \times 10^{-7}$ and that the dynamic physiological concentration ranges of ATP (= ADP-OP), ADP (= ADP- 
$\mathrm{OH})$ and $\mathrm{Pi}(=\mathrm{P}-\mathrm{OH})$ experimentally determined in mitochondria/cells is $\sim 10^{-3} \mathrm{M}, \sim 10^{-4} \mathrm{M}$, and $\sim 10^{-2} \mathrm{M}$ respectively. (Water activity can be taken as unity; otherwise its factual concentration is 50 M.) If the physiological concentration of ATP were governed by the equations (1) \& (2) and mediated by Complex V activity, the value of the equilibrium ATP levels would be-

$$
\left[\left(\mathrm{K}_{\text {eq.ATPase }}=5 \times 10^{-7}\right) \times\left(\mathrm{ADP}-\mathrm{OH}=10^{-4}\right) \times\left(\mathrm{P}-\mathrm{OH}=10^{-2}\right)\right] /\left(\mathrm{H}_{2} \mathrm{O} \sim 1\right) \approx 5 \times 10^{-13} \mathrm{M}
$$

This value afforded by equilibrium driven catalysis is tens of trillion folds lower than the observed physiological ATP levels! Therefore, the obvious question is- how can the mOxPhos reaction be catalyzed by Complex $\mathrm{V}$ and be governed by equations (1) \& (2)? An enzyme can only help the reaction attain the equilibrium status faster, but it cannot change the equilibrium position (or ratio of relative concentrations) of the given reaction. Further, however sophisticated Complex V may be, how can it achieve physiological ATP synthesis when its binding affinity for ATP is more than 10 million folds higher to that of ADP and respiring mitochondria have higher levels of ATP (Manoj et al., 2019a)? Clearly, dynamic partitioning or rapid utilization or recycling of components cannot change this "predicament" in any significant way, as the order of difference or mismatch is enormous. Regardless, let's study the turnover dynamics of ATP.

In a day, an average human body synthesizes (and consumes) about $40 \mathrm{~kg}$ of ATP (Stryer, 1981). Since the molecular mass of ATP is 507, this equals $\sim 10^{2}$ moles of ATP synthesized per day or $10^{-3}$ moles per second. If we take a person's weight as $10^{2} \mathrm{~kg}$ (equivalent to a volume of $\sim 10^{2}$ liters), then the ATP generation rate is $W=10^{-5}$ moles per second. This translates to a synthesis rate of $6 \times 10^{18}$ ATP molecules per second. In physiological steady state, since the rate of generation of $\operatorname{ATP}(W)$ is equal to the rate of hydrolysis:

$$
W=k_{\text {hydr }}[\mathrm{ATP}]
$$


From the above, the hydrolysis rate $k_{\text {hydr }}$ of ATP is determined to be $10^{-2}$ per second. That is, the average lifetime of an ATP molecule in the body is $10^{2}$ seconds (or $\sim 1.5$ minutes).

Now, In the human body, there are $\sim 3.7 \times 10^{13}$ cells (Bianconi et al., 2013). If an average human body is taken to have 100 liters, the cell density is about $\sim 3.7 \times 10^{11}$ cells per liter. Literature quotes a high variance in the mitochondrial distribution, but an average distribution of $\sim 10^{3}$ mitochondria per cell is a good statistical idea (Metzler, 2001). Via electron microscopy (Bowman et al., 1989), 3.2 x $10^{5}$ Comp. V were noted/calculated per mitochondrion. Therefore, the amount of Comp. V would be $3.2 \times 10^{5} \times 10^{3} \times 3.7 \times 10^{11}=10^{20}$ per liter. This translates to an initial enzyme concentration $E_{0}=1.7 \times 10^{-4} \mathrm{M}$. This value and the ATP hydrolysis rate calculated earlier would be used in the analysis to follow.

The elaborate reaction scheme of ATP synthesis (rate and equilibrium constants) are taken from the review by Senior (Senior, 1992). The scheme and parameters governing the distinct reaction steps of the process are shown in Figure 2 and Table 1.

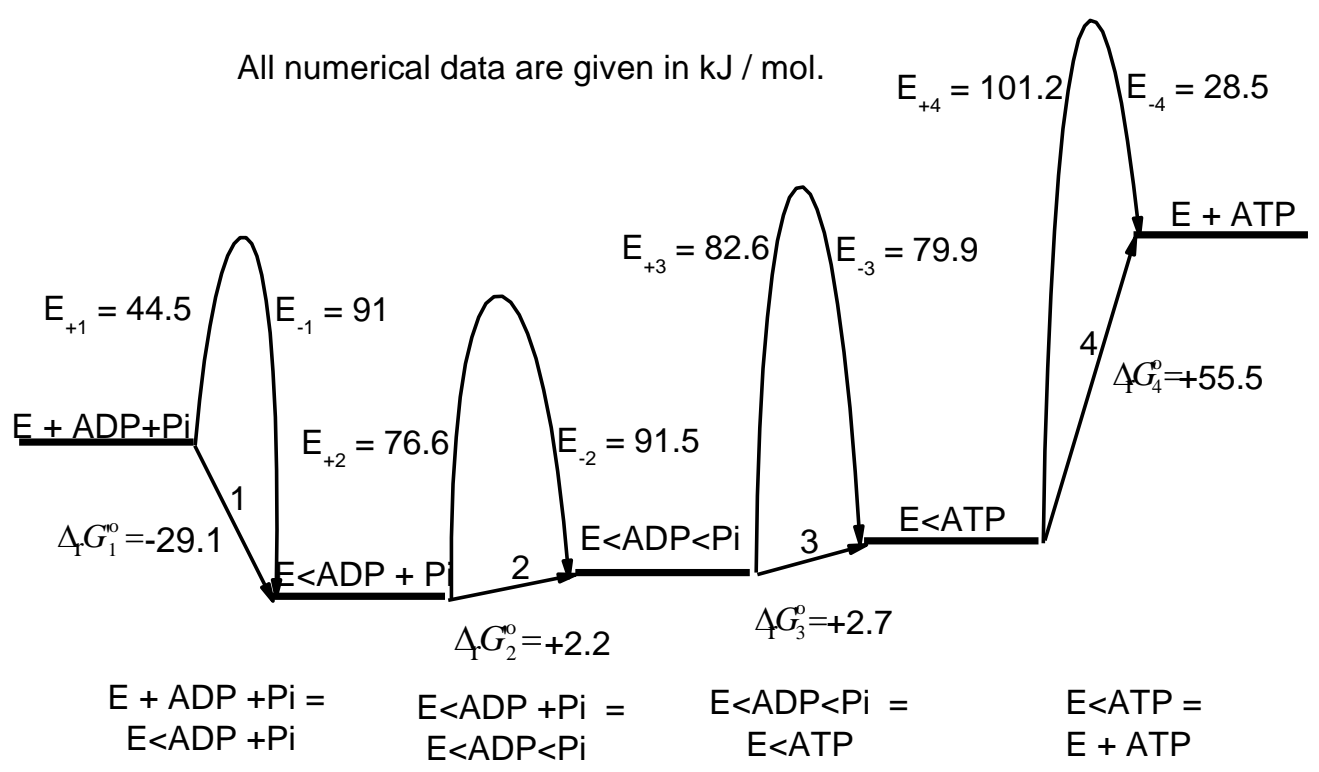


Figure 2. Reaction scheme of ATP synthesis (Senior, ): The sequentially ordered steps commence from the free enzyme $(E), A D P$ and $P i$ in milieu at the left, progressing to ADP binding (step 1), followed by ADP+Pi binding (step 2), which leads to the formation of bound ATP (step 3), and ultimately giving the free enzyme again concomitant with the release of ATP (step 4, the final one on the right). The curved arrows are used for depicting the activation energies, straight line arrows for step number (and Gibbs' energy change in that step) and the bold lines show distinct states along the reaction coordinate.

Table 1. Kinetic parameters of Complex V mediated ATP synthesis at $30^{\circ} \mathrm{C}$.

\begin{tabular}{|c|c|c|c|c|c|c|c|}
\hline \multicolumn{2}{|c|}{$\begin{array}{l}\text { Reactions rate } \\
\text { constants }\end{array}$} & \multicolumn{2}{|c|}{$\begin{array}{l}\text { Ectivation energy*, } \\
\mathrm{kJ} / \mathrm{mol}\end{array}$} & \multicolumn{2}{|c|}{ Equilibrium constant } & \multicolumn{2}{|c|}{$\begin{array}{l}\text { Gibbs energy } \\
\text { change, } \mathrm{kJ} / \mathrm{mol}\end{array}$} \\
\hline Definition & Value & Definition & Value & Definition & Value & Definition & Value \\
\hline $\mathrm{k}_{+1}\left(\mathrm{M}^{-1} \mathrm{~s}^{-1}\right)$ & $1.8 \times 10^{2}$ & $\overline{E_{+1}}$ & 44.5 & $\mathrm{~K}_{1}$ & $1.1 \times 10^{5}$ & $\Delta G^{\prime \circ}$ & -201 \\
\hline $\mathrm{k}_{-1}\left(\mathrm{~s}^{-1}\right)$ & $1.6 \times 10^{-3}$ & $\mathrm{E}_{-1}$ & 91 & & & $\Delta_{\mathrm{r}} \mathrm{G}_{1}$ & -29.1 \\
\hline $\mathrm{k}_{+2}\left(\mathrm{M}^{-1} \mathrm{~s}^{-1}\right)$ & $4.8 \times 10^{-4}$ & $\overline{\mathrm{E}_{+2}}$ & 76.6 & $\mathrm{~K}_{2}$ & 0.4 & & +2.2 \\
\hline $\mathrm{k}_{-2}\left(\mathrm{~s}^{-1}\right)$ & $1.2 \times 10^{-3}$ & $\mathrm{E}_{-2}$ & 91.5 & & & $\Delta_{\mathrm{r}} G_{2}$ & \\
\hline $\mathrm{k}_{+3}\left(\mathrm{~s}^{-1}\right)$ & 0.043 & $\mathrm{E}_{+3}$ & 82.6 & $\mathrm{~K}_{3}$ & 0.3 & $A G^{10}$ & 77 \\
\hline $\mathrm{k}_{-3}\left(\mathrm{~s}^{-1}\right)$ & 0.12 & $\mathrm{E}_{-3}$ & 79.9 & & & & +2.7 \\
\hline $\mathrm{k}_{+4}\left(\mathrm{~s}^{-1}\right)$ & $2.5 \times 10^{-5}$ & $E_{+4}$ & 101.2 & $\mathrm{~K}_{4}$ & $2.5 \times 10^{-10}$ & & \\
\hline $\mathrm{k}_{-4}\left(\mathrm{M}^{-1} \mathrm{~s}^{-1}\right)$ & $1.1 \times 10^{5}$ & $\mathrm{E}_{-4}$ & 28.5 & & & $\Delta_{\mathrm{r}} \mathrm{C}$ & +55.5 \\
\hline
\end{tabular}

*The activation energies were calculated from the assumption of reaction rate constants in the form: for monomolecular reactions $k_{m}=10^{13} \cdot \exp (-E / R T) \mathrm{s}^{-1}$ and for bimolecular $k_{b}=10^{10} \cdot \exp (-E / R T) 1 /(\mathrm{mol} . \mathrm{s})$.

Let us now consider the individual steps of the reaction scheme.

\section{Step 1}

Note that the initial concentration of ADP is significantly higher than the concentration of synthases. The concentration of ADP almost does not change when E<ADP is formed. For the equilibrium constant of the first reaction, we obtain-

$$
K_{1}^{\prime}=1.1 \cdot 10^{5}=\frac{[\mathrm{E}<\mathrm{ADP}]}{[\mathrm{E}] \cdot[\mathrm{ADP}]}=\frac{[\mathrm{E}<\mathrm{ADP}]}{\left(E_{0}-[\mathrm{E}<\mathrm{ADP}]\right) \cdot[\mathrm{ADP}]}
$$


From the above, we get-

$$
\frac{[\mathrm{E}<\mathrm{ADP}]}{E_{0}}=\frac{K_{1}^{\prime} \cdot[\mathrm{ADP}]}{1+K_{1}^{\prime} \cdot[\mathrm{ADP}]}=\frac{100}{1+100} \approx 1
$$

This means that all available sites are occupied by ADP molecules and $[\mathrm{E}<\mathrm{ADP}] \approx E_{0}$, where $E_{0}$ is the initial concentration of the enzyme $\mathrm{E}$. The attachment rate of ADP to $\mathrm{E}$ at the initial instant is $3 \times 10^{-5} \mathrm{~mol} /(1 . \mathrm{s})$ and the ADP molecules occupy all available places on the enzyme $\mathrm{E}$ in $~ 30$ seconds.

\section{Step 2.}

The equilibrium in the second reaction is described by the expression-

$$
K_{2}^{\prime}=0,41=\frac{[\mathrm{E}<\mathrm{ADP}<\mathrm{Pi}]}{[\mathrm{E}<\mathrm{ADP}] \cdot[\mathrm{Pi}]}
$$

From the above, we get-

$$
\frac{[\mathrm{E}<\mathrm{ADP}<\mathrm{Pi}]}{[\mathrm{E}<\mathrm{ADP}]}=0,4 \cdot[\mathrm{Pi}]=0,4 \cdot 10^{-3}=4 \cdot 10^{-4}
$$

Hence $[\mathrm{E}<\mathrm{ADP}<\mathrm{Pi}]=4 \cdot 10^{-4}[\mathrm{E}<\mathrm{ADP}] \approx 4 \cdot 10^{-4} E_{0}=4 \cdot 10^{-4} \cdot 1.7 \cdot 10^{-4}=6.8 \cdot 10^{-8} \mathrm{M}$

The concentration of $\mathrm{Pi}$ in these reactions varies slightly, since $[\mathrm{Pi}]>>\mathrm{E}_{0}$. Therefore, the second reaction's rate is: $\mathrm{W}_{+2}=\mathrm{k}_{+2} \cdot[\mathrm{E}<\mathrm{ADP}] \cdot[\mathrm{Pi}]=4.8 \times 10^{-4} \times 1.7 \times 10^{-4} \times 10^{-4}=8 \times 10^{-11} \mathrm{~mol} /(1 \cdot \mathrm{s})$. The rate of this reaction is very low in order to ensure a high reaction rate of ATP formation in solution and reach a concentration $10^{-3} \mathrm{M}$.

\section{Step 3.}

The equilibrium in the third reaction is described by the expression- 


$$
0.34=\frac{[\mathrm{E}<\mathrm{ATP}]}{[\mathrm{E}<\mathrm{ADP}<\mathrm{Pi}]}=\frac{[\mathrm{E}<\mathrm{ATP}]}{4 \cdot 10^{-4} E_{0}}
$$

So, $[\mathrm{E}<\mathrm{ATP}]=1.4 \cdot 10^{-4} E_{0}=1.4 \cdot 10^{-4} \cdot 1.7 \cdot 10^{-4}=2.4 \cdot 10^{-8} \mathrm{M}$.

The rate of the third reaction, $W_{+3}=0.043 \times 2.4 \cdot 10^{-8}=10^{-9} \mathrm{~mol} /(1 \cdot \mathrm{s})$.

\section{Step 4.}

The rate of formation of ATP in solution is-

$$
\frac{d[\mathrm{ATP}]}{d t}=k_{+4} \cdot[\mathrm{E}<\mathrm{ATP}]=2.5 \cdot 10^{-5} \cdot 2.4 \cdot 10^{-8}=6 \cdot 10^{-13} \mathrm{~mol} /(1 \cdot \mathrm{s})
$$

Since ATP hydrolysis occurs with a rate constant of $0.01 \mathrm{~s}^{-1}$, then compensating this with respect to the equilibrium ATP concentration, we obtain the "original" ATP concentration as $6 \cdot 10^{-11} \mathrm{M}$. Even this value is only two orders of magnitude higher than the theoretical equilibrium value $5 \mathrm{x}$ $10^{-13} \mathrm{M}$ and still much lower the factual physiological ATP concentration of $10^{-3} \mathrm{M}$.

The exhaustive analyses above shows that Complex V (with the known/published constants for ATPsynthase) cannot perform the physiologically observed higher rates of ATP synthesis. Even if the ATP is removed with the highest efficiency from synthase (as Paul Boyer suggests), it is impossible to obtain a high rate of ATP formation in solution, as the slow stages of the second and third reactions are limiting. Similar results also arise in the calculation with a different set of reaction constants published (Cunningham \& Richard, 1988). Complex V, being a catalyst, can provide only the maximum concentration permitted by thermodynamics/equilibrium. 
What is the actual "thermodynamic" role of protons in ATP synthesis or thermogenesis? How is the physiological kinetics of ATP-synthesis explained?

Unlike the "closed pot" purview (which is required for the buld-up of proton-differential) of chemiosmosis, under the murburn explanation for mOxPhos, ATP synthesis is essentially an "open pot" process. This is because mOxPhos is a proton-deficient scheme, as oxidation of NADH gives two electrons but only one proton. Also, the Keilin-Mitchell-Boyer scheme does not chemically connect NADH oxidation directly with ATP synthesis. Further, in the chemiosmosis scheme, water formation is through two distinct and disconnected means (via ETC and via Complex V) and thermogenesis results from yet another thermodynamically unviable explanation (Manoj, 2017; Manoj, 2018a). In contrast, the murburn scheme directly connects NADH oxidation with ATP synthesis, water formation and thermogenesis via diffusible reactive oxygen species (DROS). In the murburn purview, protons are required in the steady state conditions, and this must be replenished from the cytoplasm and the proton-utilization reaction occurs in IMS or matrix. This is also because only the formation of water satisfies the "thermodynamic viability" criteria in terms of bond energetics. That is,. the O-H bond has $\sim 467$ $\mathrm{kJ} / \mathrm{mol}$ (whether via the formation of ATP or through reactions of DROS amongst themselves leading to thermogenesis), and this is one of the major contributors on the right hand side of the reaction equation. Further, the outward movement of the newly formed water molecule via colligative pressures exerts a thermodynamic pull (right-ward) on the oxygen-superoxide equilibrium, thereby depleting more NADH/oxygen and consequentially synthesizing more ATP or generating heat. It is evident from the scheme shown in Figure 3 that DROS is the sole and main-point fulcrum for ATP-synthesis, water formation and heat generation (Manoj, 2017; 
Manoj et al., 2018a; Manoj 2019a). Further, protons also influence the intricate reaction equilibriums and affect kinetics within matrix, as highlighted in our earlier works (Manoj et al., 2018a).

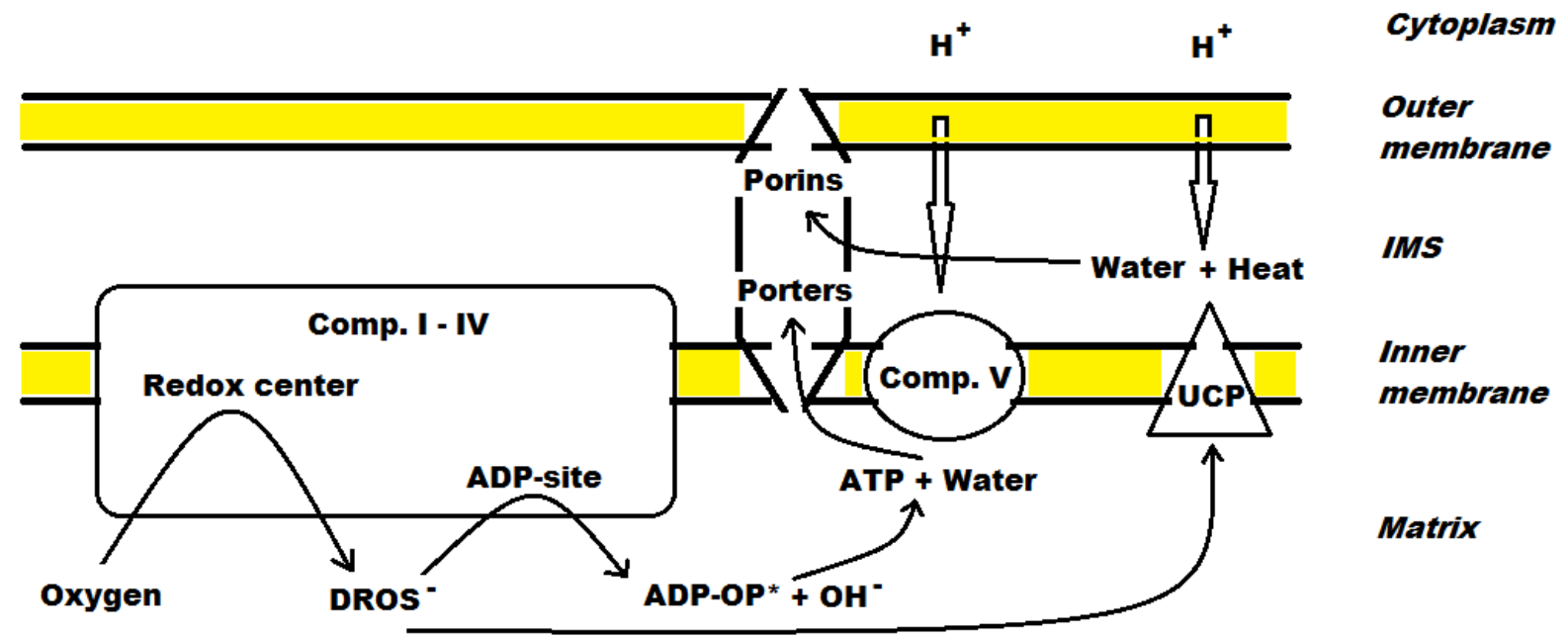

Figure 3: The overall scheme of ATP-synthesis, water formation and thermogenesis in the murburn scheme is mediated via DROS (diffusible reactive oxygen species). The four components on the right (porters, porins, Comp. V and UCP) are involved only in steady state steps whereas Complexes I through IV are directly involved in ATP synthesis. Heat conservation by the ensconcing lipid membranes (more effective insulators) is efficient when DROS are leaked into the IMS via UCP (which leads to lowering of TMP as the negative charges are more quickly neutralized by cytoplasmic protons, leading to water formation).

The steady-state mass-charge balanced oxygen-centric murburn equation (wherein the reaction/catalysis is mediated via DROS) is-

$\mathrm{NADH}+\mathrm{ADP}-\mathrm{OH}+\mathrm{P}-\mathrm{OH}+\mathrm{O}_{2}+\mathrm{H}^{+}$cytoplasm $\rightarrow \mathrm{NAD}^{+}+\mathrm{ADP}-\mathrm{OP}+\mathrm{H}_{2} \mathrm{O}+\mathrm{H}_{2} \mathrm{O}_{2}---(7)$

For this equation, the equilibrium expression would be-

$\mathrm{K}_{\text {eq.murburn }}^{\prime}=\left(\left[\mathrm{NAD}{ }^{+}\right][\mathrm{ADP}-\mathrm{OP}]\left[\mathrm{H}_{2} \mathrm{O}\right]\left[\mathrm{H}_{2} \mathrm{O}_{2}\right]\right) /\left([\mathrm{NADH}][\mathrm{ADP}-\mathrm{OH}][\mathrm{P}-\mathrm{OH}]\left[\mathrm{O}_{2}\right]\left[\mathrm{H}^{+}\right]\right)---(8)$

The equilibrium constant for the above reaction can be calculated from the known experimental values. With the following considerations- $\left(\left[\mathrm{NAD}^{+}\right] /[\mathrm{NADH}]=\sim 10^{2}\right)$, as $\mathrm{NAD}^{+} / \mathrm{NADH}$ ratios vary between 7 to 8 in the mitochondria and range between 60 to 700 in cytoplasm and since 
both these pools are in equilibrium, a conservative value of $10^{2}$ is appropriate; $\left[\mathrm{H}_{2} \mathrm{O}_{2}=10^{-6} \mathrm{M}\right]$ ), as DROS production/detection may range between 0.1 to $10 \%$ of the approximate $\left[\mathrm{O}_{2}=10^{-4} \mathrm{M}\right]$; $\left[\mathrm{H}^{+}=10^{-7} \mathrm{M}\right]$ and $\mathrm{H}_{2} \mathrm{O}$ activity $=10^{0} ;\left[\mathrm{ADP}-\mathrm{OP}=10^{-3} \mathrm{M}\right] ;\left[\mathrm{ADP}-\mathrm{OH}=10^{-4} \mathrm{M}\right] ;\left[\mathrm{P}-\mathrm{OH}=10^{-2}\right.$ $\mathrm{M}]$;

$$
\mathrm{K}_{\text {eq.murburn }}^{\prime}=\left(10^{2} \times 10^{-3} \times 10^{0} \times 10^{-6}\right) /\left(10^{-4} \times 10^{-2} \times 10^{-4} \times 10^{-7}\right)=10^{10} \mathrm{M}^{-1}
$$

The dimensionless matrix-phase equilibrium constant can be derived by multiplying the

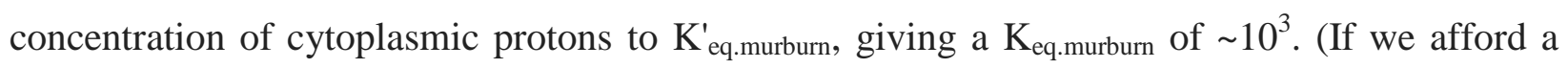
higher activity for water, the equilibrium constant would be even higher!) Therefore, the murburn reaction equilibrium constant value is higher than rotary ATPase catalysis value by $>10^{10}$ ! Now (by substituting $\mathrm{K}_{\text {eq.murburn }}$ value in equation (1)), we can easily rationalize the production of physiological levels of ATP $\left(10^{-3} \mathrm{M}\right)$ from the starting concentrations of $10^{-4} \mathrm{M}$ $\mathrm{ADP}$ and $10^{-2} \mathrm{M}$ Pi. The treatment above is conclusive theoretical evidence for the physiological linking of NADH oxidation and ATP synthesis via DROS. The requirement of cytoplasmic protons in steady-state explains the earlier findings supporting the Keilin-Mitchell-Boyer model.

To summate, TMP is a consequence of mitochondrial oxidative phosphorylation (occurring within the matrix), and not the cause. The redox potential of metabolically generated NADH is directly used for a fast and efficient ATP-synthesis in a simple chemical reaction scheme (and these processes also result in thermogenenesis), all via the intermediacy of DROS. This statement is further corroborated by the consideration that murburn concept also explains the acutely lethal effect of low amounts of cyanide (a known mOxPhos inhibitor, Manoj et al., 2019b) and successfully predicts several tens of outcomes in mitochondrial physiology (Manoj et al., 2019c). 
Acknowledgments \& Disclaimers: This work was powered by Satyamjayatu: The Science \&

Ethics Foundation. The authors do not have any conflict of interests to disclose.

\section{References}

Alberty RA. Thermodynamics of Biochemical Reactions, USA: Wiley (2003).

Bazhin NM. Gibbs energy role in fresh and salt water mixing. Desalination 2015a, 365, 343-346.

Bazhin NM. Heat and work of the chemical systems. J Eng Thermophys 2018, 27, 72 - 81. doi: 10.1134 / S1810232818010083

Bazhin NM. Systems with a concentration gradient have no potential energy but can do useful work. Biophysics 2015b, 1, 160-161. doi: 10.1134/S0006350915010054

Bianconi E, Piovesan A, Facchin F, Beraudi A, Casadei R, Frabetti F, Vitale L, Pelleri MC, Tassani S, Piva F, Perez-Amodio S, Strippoli P, Canaider S, An estimation of the number of cells in the human body. Ann Hum Biol 2013; 40, 463-471.

Bowman BJ, Dschida WJ, Harris T, Bowman EJ. The vacuolar ATPase of Neurospora crassa contains an F1-like structure. J Biol Chem 1989, 264, 15606-15612.

Crabtree B, Taylor DJ. Thermodynamics and matter exchange in "Biochemical thermodynamics" ed. by M. N. Jones, Elsevier, Amsterdam (1979).

Cunningham D, Cross RL. Catalytic site occupancy during ATP hydrolysis by mf1-ATPase: evidence for alternating high affinity sites during steady-state turnover. J Biol Chem 1988, . 263, 18850-18856.

Deng Y, Stratt RM. Vibrational energy relaxation of polyatomic molecules in liquids: The solvent's perspective. J Chem Phys 2002, 117(4), 1735-1749. doi:10.1063/1.1489417

Hubley MJ, Bruce RL, Timothy SM. The effects of temperature, $\mathrm{pH}$, and magnesium on the diffusion coefficient of ATP in solutions of physiological ionic strength. Biochim Biophys Acta 1996, 1291, 115-121.

Manoj KM, Parashar A, Jacob VD, Ramasamy S. Aerobic Respiration: Proof of concept for the murburn perspective. J Biomol Str Dynam 2019a. doi: 10.1080/07391102.2018.1552896

Manoj KM. Aerobic respiration: Criticism of the proton-centric explanation involving rotary ATP synthesis, chemiosmosis principle, proton pumps and electron transport chain. Biochem Insights 2018. doi:10.1177/1178626418818442 
Manoj KM. Chemiosmosis principle versus murburn concept: Why do cells need oxygen? OSF preprints 2019b. doi: 10.31219/osf.io/3jq8m

Manoj KM. Debunking chemiosmosis and proposing murburn concept as the explanation for cellular respiration. Biomed Rev 2017; 28:35-52. doi:10.14748/bmr.v28.4450

Manoj KM. Gideon DA, Jacob VD. Murburn scheme for mitochondrial thermogenesis. Biomed Rev 2018a; 29, 73-82. doi: 10.14748/bmr.v29.5852

Manoj KM. The ubiquitous biochemical logic of murburn concept. Biomed Rev 2018b; 29, 8997. doi: 10.14748/bmr.v29.5854

Manoj KM, Ramasamy S, Parashar A, Soman V, Pakshirajan K. Murburn concept explains the acutely lethal effect of cyanide. bioRxiv preprint 2019b. doi: https://doi.org/10.1101/555888

Manoj, KM, Soman V, Jacob VD, Parashar A, Gideon DA, Kumar M, Manekkathodi A, Ramasamy S, Pakshirajan K. Chemiosmosis principle versus murburn concept: Why do cells need oxygen? Deducing the underpinnings of aerobic respiration by mechanistic predictability. OSF Preprints, 2 May 2019. doi: 10.31219/osf.io/3jq8m

Metzler D. Biochemistry, Second edition, Elsevier Academic Press (2001).

Nelson D, Cox M. Lehninger; Principles of Biochemistry, Fifth edition, Freeman, NY (2008).

Prigogine I, Defay R. Chemical Thermodynamics, Longman, London (1954).

Rubin AB. Biophysics, v. II, Moscow (2013, in Russian).

Senior AE. Catalytic sites of Escherichia coli F $_{1}$-ATPase. J Bioenerg Biomemb 1992, 24, 479484.

Strong LE, Halliwell HF. An alternative to free energy for undergraduate instruction J Chem Educ 1970, 47, 347-352.

Stein LR, Imai S-I. The dynamic regulation of NAD metabolism in mitochondria. Trends Endocrinol Metab 2012, 23, 420-428. doi: 10.1016/j.tem.2012.06.005

Stryer L. Biochemistry, Freeman, San Francisco (1981). 